
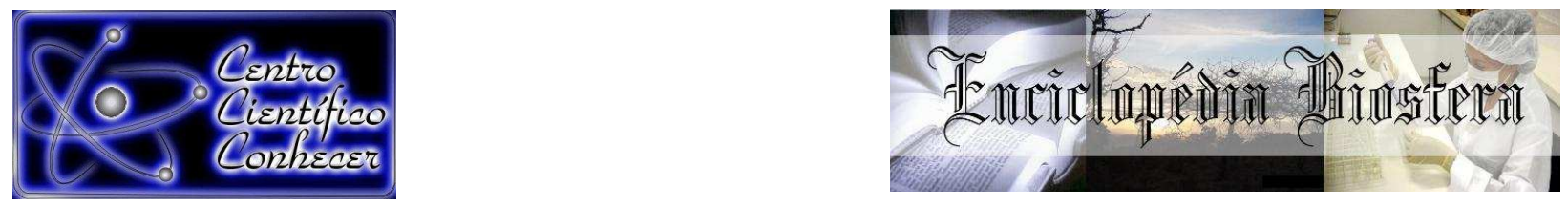

\title{
MÉTODOS ECODOPPLERCARDIOGRÁFICOS PARA AVALIAÇÃO DA FUNÇÃO DIASTÓLICA EM PEQUENOS ANIMAIS
}

\author{
Ana Paula Araujo Costa ${ }^{1}$; Priscilla Regina Nasciutti ${ }^{1}$; Marcelo Borges dos Santos \\ Junior $^{2}$; Thaiz Krawczyk Santos ${ }^{3}$; Rosângela de Oliveira Alves Carvalho ${ }^{4}$ \\ ${ }^{1}$ Doutoranda, Escola de Veterinária e Zootecnia da Universidade Federal de Goiás, \\ Goiânia, Goiás, Brasil. e-mail: hananinha@gmail.com \\ ${ }^{2}$ Mestre, Médico Veterinário Autônomo, Goiânia, Goiás, Brasil. \\ ${ }^{3}$ Graduanda em Medicina Veterinária, Escola de Veterinária e Zootecnia da \\ Universidade Federal de Goiás, Goiânia, Goiás, Brasil. \\ ${ }^{4}$ Doutora, Docente Escola de Veterinária e Zootecnia da Universidade Federal de \\ Goiás, Goiânia, Goiás, Brasil
}

Recebido em: 08/09/2015 - Aprovado em: 14/11/2015 - Publicado em: 01/12/2015

DOI: http://dx.doi.org/10.18677/Enciclopedia_Biosfera_2015_195

\begin{abstract}
RESUMO
A ecodopplercardiografia é uma ferramenta de diagnóstico complementar muito importante na clínica cardiovascular dos pequenos animais e há algum tempo já vem sendo utilizada rotineiramente. Uma das principais informações obtidas com essa avaliação é a quantificação da função sistólica do ventrículo esquerdo, por meio do cálculo das frações de ejeção e encurtamento. No entanto, a disfunção diastólica precede a sistólica em algumas enfermidades como por exemplo a cardiomiopatia dilatada. Considerando que a quantificação da função diastólica ainda não é rotineira em medicina veterinária e que seu comprometimento acarreta graves consequências clínicas para os pacientes, essa revisão objetivou discorrer sobre os métodos ecodopplercardiográficos de avaliação da função diastólica do ventrículo esquerdo de pequenos animais e relatar quais os achados mais frequentes nas principais enfermidades que acometem o sistema cardiovascular.
\end{abstract}

PALAVRAS-CHAVE: cães, ecocardiograma, função ventricular, gatos

\section{ECHODOPPLERCARDIOGRAPHIC METHODS TO ASSESS DIACTOLIC FUNCTION OF SMALL ANIMALS}

\begin{abstract}
Doppler echocardiography is a very important complementary diagnostic tool in cardiovascular clinic of small animals and is already being used routinely for some time. One of the main information obtained from this is the quantification of systolic function of the left ventricle, through the calculation of ejection and shortening fractions. However, diastolic dysfunction precedes systolic dysfunction in some diseases such as dilated cardiomyopathy. Considering that quantification of diastolic function is still not a routine in veterinary medicine and that their commitment has serious clinical consequences for patients, this review aimed to discuss the Doppler echocardiographic methods for assessing left ventricular diastolic function in small


animals and report the findings in major diseases that affect the cardiovascular system.

KEYWORDS: echocardiogram, cats, dogs, ventricular function,

\section{INTRODUÇÃO}

A função ventricular sistólica é avaliação rotineira em qualquer animal submetido ao exame ecodopplercardiográfico. As mensurações tradicionalmente feitas para acessar a função sistólica do ventrículo esquerdo (VE) são as taxas de fração de ejeção (FE) e fração de encurtamento (FS) (BONAGURA \& SCHOBER, 2009), sendo a disfunção sistólica caracterizada por bombeamento sanguíneo inadequado e redução da fração de ejeção (BOON, 2011).

No entanto, em muitas enfermidades cardiovasculares, a disfunção diastólica precede a sistólica, como reportado em pacientes caninos portadores de cardiomiopatia dilatada (O`SULLIVAN et al., 2007). A função diastólica do ventrículo esquerdo compreende a capacidade desse em acomodar um adequado enchimento volumétrico para manter o débito cardíaco apropriado às necessidades basais do organismo. A disfunção diastólica dessa forma, resulta em importantes consequências clínicas e em muitos casos, como reportado nos seres humanos, ela ocorre de forma silenciosa, sendo um achado incidental em aproximadamente 25 a 30\% dos indivíduos acima de 45 anos (LESTER et al., 2008).

Outro estudo conduzido em seres humanos demonstrou que a disfunção diastólica, caracterizada pelo aumento da pressão de enchimento do ventrículo esquerdo, aumenta drasticamente o risco de complicações cardiovasculares ou cardíacas (KUKZETSOVA et al. 2014). Similarmente em cães que apresentam severa regurgitação mitral, condição comum em paciente portadores de mixomatose da válvula mitral (MVM), achados ecodopplercardiográficos sugestivos de aumento da pressão de enchimento do ventrículo esquerdo (VE) são relatados como preditores de insuficiência cardíaca (BONAGURA \& SCHOBER, 2009).

Porém a utilização da avaliação da função diastólica na rotina clínica cardiovascular requer conhecimento prévio dos métodos disponíveis. Portanto, com essa revisão objetivou-se discorrer sobre os métodos ecodopplercardiográficos de avaliação da função diastólica do ventrículo esquerdo de pequenos animais e relatar quais os achados mais frequentes nas principais enfermidades que acometem 0 sistema cardiovascular.

\section{MENSURAÇÃO DA DISFUNÇÃO DIASTÓLICA}

A mensuração tradicional da função diastólica inclui a análise dos fluxos transmitral e das veias pulmonares, obtidas no exame ecodopplercardiográfico tradicional. O Doppler tecidual é outro método ecodopplercardiográfico incorporado a avaliação da função diastólica, o qual demonstra as alterações de velocidade de movimentação do músculo cardíaco (LO \& THOMAS, 2010; BONAGURA \& FUENTES, 2015).

No intuito de simplificar a compreensão da avalição do estudo ecodopplercardiográfico tradicional, bem como dos gráficos de movimentação do miocárdio, obtidos na avaliação Doppler tecidual, primeiramente serão descritas as fases da diástole e posteriormente os fluxos e análise Doppler tecidual, que permitem a graduação da disfunção diastólica. 


\section{FASES DA DIÁSTOLE}

Analisando isoladamente o ventrículo esquerdo, a diástole é definida como o período compreendido entre o fechamento da válvula aórtica e abertura e fechamento da válvula mitral. A sequência de eventos que compõem esse período pode ser dividida em quatro fases: tempo de relaxamento isovolumétrico (TRIV); fase rápida de enchimento ventricular, diástase e fase lenta de enchimento ventricular ou contração atrial (PARASHAR, 2009; BOON, 2011).

Após o pico sistólico a pressão intraventricular cai e, uma vez que essa cai abaixo da pressão aórtica, ocorre o fechamento da valva semilunar e inicia-se a fase de relaxamento isovolumétrico. O TRIV começa, portanto, com o fechamento da válvula aórtica e termina quando há a abertura da válvula mitral, não ocorrendo durante essa fase mudanças no volume do ventrículo esquerdo e permanecendo todas a válvulas fechadas, porém a pressão intraventricular continua a cair e o miocárdio relaxa. Simultaneamente, está ocorrendo aumento da pressão atrial devido ao preenchimento do átrio esquerdo pelas veias pulmonares, estabelecendose dessa forma um gradiente pressórico atrioventricular (PARASHAR, 2009; BOON, 2011).

Quando a pressão atrial supera a intraventricular ocorre a abertura da válvula mitral e com essa inicia-se a fase de preenchimento rápido do ventrículo esquerdo. Estima-se que $80 \%$ do preenchimento do VE ocorre nessa fase, fazendo com que haja aumento da pressão intraventricular e, à medida que a pressão no VE se equilibra ou mesmo supera a do átrio esquerdo, irá ocorrer uma desaceleração do fluxo mitral, fenômeno esse conhecido como tempo de desaceleração (DT). Durante o período de equilíbrio pressórico atrioventricular, ocorre movimento passivo de sangue do átrio esquerdo para o ventrículo esquerdo, sendo essa fase denominada de diástase (PARASHAR, 2009; BOON, 2011).

A fase de enchimento rápido ou diástase depende da perfeita interação entre o fenômeno de sucção ventricular, que ocorre devido ao seu relaxamento ativo, e de suas propriedade viscoelástica passivas ou complacência. Após o equilíbrio pressórico entre o átrio e ventrículo esquerdos, o átrio irá agir como uma bomba, contribuindo com aproximadamente $20 \%$ do enchimento ventricular, iniciando-se a fase de contração atrial ou enchimento ventricular lento ou tardio. Essa contração atrial irá acelerar o fluxo transmitral, porém não na mesma proporção em que ocorre durante a fase rápida de enchimento ventricular, no começo da diástole (PARASHAR, 2009; BOON, 2011).

\section{ECODOPPLERCARDIOGRAFIA CONVENCIONAL E DOPPLER TECIDUAL}

\section{Fluxo transmitral}

O princípio Doppler, descrito por Christian Johann Doppler, diz que todas as ondas, sejam sonoras, luminosas, ou outras, mudam em comprimento de onda quando ocorre mudança na posição entre a fonte da onda e o receptor da onda. Aplicando-se esse princípio ao estudo do fluxo vascular, pode-se dizer que quando as ondas sonoras transmitidas pelo transdutor do equipamento ultrassonográfico atingem as células sanguíneas em movimento, a onda que retorna ao transdutor sofre mudanças em sua frequência. Em outras palavras, células sanguíneas movimentando-se em direção ou transdutor irão refletir um número maior de ondas e, consequentemente a frequência da onda recebida pelo transdutor será maior que a transmitida. Em contrapartida, células sanguíneas movimentando-se na direção contraria irão diminuir a freqüência das ondas recebidas pelo transdutor. Essas 
mudanças de frequência são calculadas (Sinal Doppler - Doppler shift) e representadas de diferentes formas, espectrais ou coloridas na tela do equipamento (BOON, 2011).

As informações de direção de fluxo sanguíneo são traduzidas em escala de cores (Doppler colorido) e/ou graficamente (Doppler pulsado) na imagem ultrassonográfica. Foi convencionado que fluxos que seguem em direção ao transdutor são demonstrados em vermelho e fluxos que seguem em direção contrária são representados em azul (STALMANS et al., 2011). Graficamente, os fluxos em direção ao transdutor aparecem acima da linha de base e os na direção contrária, abaixo da linha de base. $O$ eixo das abscissas $(x)$, na escala gráfica do Doppler pulsado, representa o tempo decorrido, expresso em milissegundos (ms), e o eixo das ordenadas (y) representa a velocidade registrada, expressa em metros por segundo (m/s) (BOON, 2011).

Por meio da avaliação do padrão do fluxo de entrada do ventrículo esquerdo, ou fluxo transmitral, ou mitral, obtém-se informações sobre as fases de enchimento ventricular, sendo esse fluxo diretamente proporcional ao gradiente pressórico atrioventricular (LESTER et al., 2008). O fluxo transmitral é positivo à avaliação Doppler pulsado e apresenta dois picos de velocidade, ou duas ondas, E e $A$, assemelhando-se a uma letra $M$ quando os pacientes não estão em taquicardia. Pacientes que apresentam frequência cardíaca superior a 125bpm podem apresentar sobreposição, não sendo possível diferenciar as duas ondas quando a frequência supera os 200bpm (BOON, 2011).

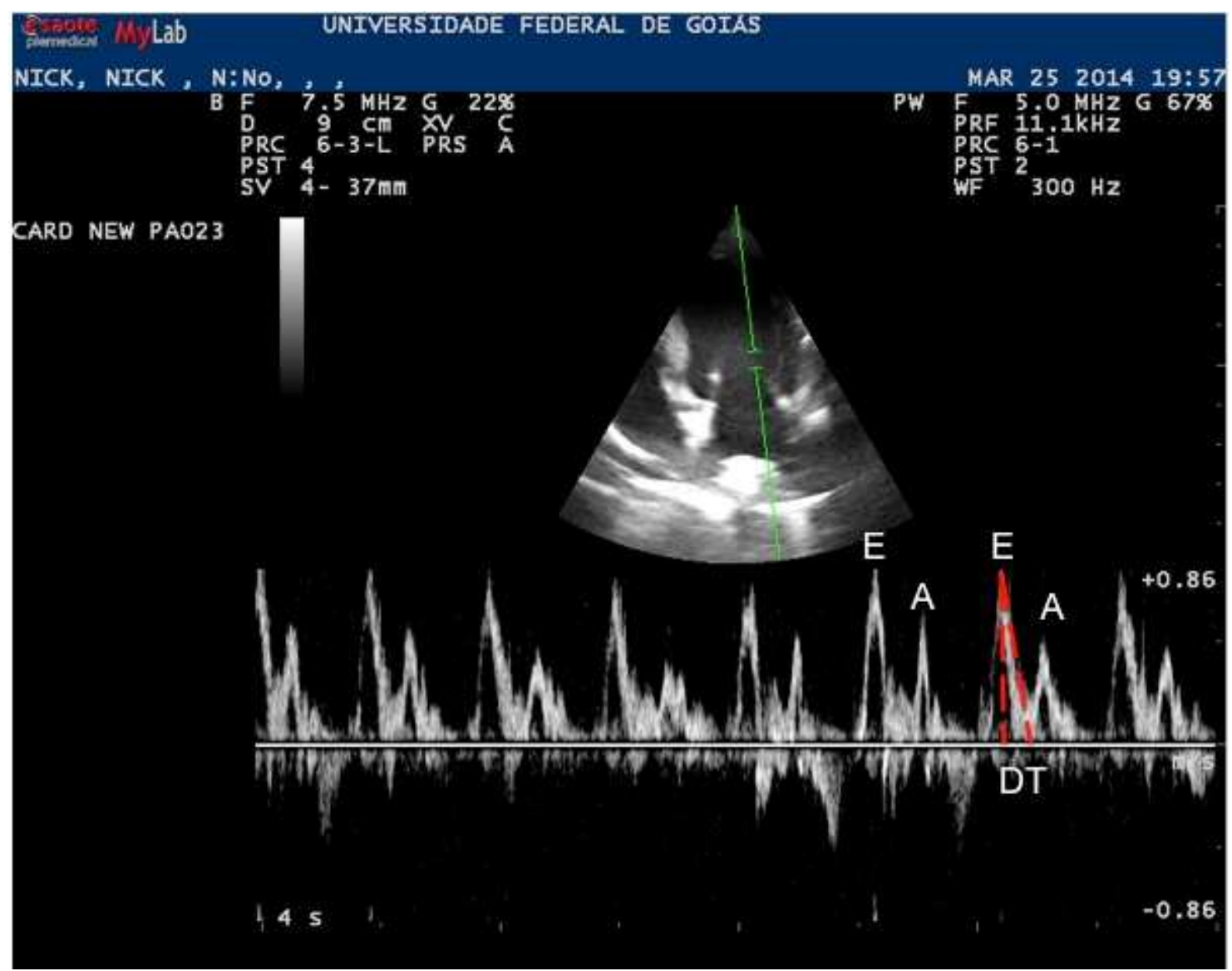

FIGURA 1. Fluxo transmitral normal de um cão, identificando picos de velocidades, das ondas $E$ e A, e tempo de desaceleração da onda E (DT). Fonte: arquivo Serviço de Cardiologia Veterinária do Hospital Veterinário da UFG, 2014. 
O pico de velocidade, ou onda $E$, representa a fase rápida de enchimento ventricular e é influenciada pelo gradiente pressórico atrioventricular. O gradiente pressórico por sua vez, é influenciado por uma diversidade de fatores incluindo a pressão do átrio esquerdo, relaxamento e complacência do ventrículo esquerdo e pré-carga. $O$ enchimento ventricular durante a contração atrial é representado pela onda ou pico de velocidade A e é basicamente influenciado pela complacência do ventrículo esquerdo e contratilidade do átrio esquerdo (LESTER et al., 2008; NAGUEH et al., 2009; BOON, 2011; BONAGURA \& FUENTES, 2015) (Figura 1).

Como a maior parte do enchimento ventricular e esvaziamento do átrio esquerdo ocorrem durante a fase de enchimento rápido, 0 átrio encontra-se relativamente vazio ao final da diástole. Assim sendo, o gradiente pressórico atrioventricular é pequeno ao final da diástole, resultando em uma onda $A$ de baixa velocidade. Em contrapartida, a onda $E$ é de alta velocidade uma vez que o gradiente pressórico atrioventricular nessa fase é maior (PARASHAR, 2009). Em outras palavras, em animais onde a função diastólica está preservada, o pico de velocidade $E$, correspondente a fase de enchimento ventricular rápido, deve ser maior que o pico de velocidade $A$, oriundo da contração atrial (BOON, 2011) .

$A$ razão entre 0 valores dos picos de velocidade $E$ e $A$ deve ser sempre maior que um, em animais normais. No entanto, as condições de taquicardia ou bradicardia irão fazer com que a razão $\mathrm{E}: \mathrm{A}$ seja próxima a um. $\mathrm{O}$ aumento do volume sanguíneo no átrio de pacientes bradicárdicos faz com que a velocidade do fluxo transmitral oriundo da contração atrial seja maior, aumentando o pico da onda A, diminuindo a razão E:A. Já em pacientes taquicárdicos, irá ocorrer um diminuição da onda $E$ devido a diminuição do volume durante a fase de enchimento rápido e aumento do fluxo associado a contração atrial (BOON, 2011). A fusão das ondas $\mathrm{E} e$ A inviabiliza a mensuração do DT, no entanto o tempo de relaxamento isovolumétrico parece não sofrer alterações (NAGUEH et al., 2009).

$O$ tempo de desaceleração da onda $E$ corresponde ao intervalo entre 0 pico de velocidade $\mathrm{E}$ até o momento em que a onda retorna a linha de base. Esse intervalo, ou tempo de desaceleração, representa a fase em que ocorre equilíbrio entre as pressões do átrio e ventrículo esquerdos, ou diástase, e acontece entre a fase de enchimento rápido e a de contração atrial (Figura 1). Quando o gradiente pressórico atrioventricular diminui ou aumenta, durante a fase de enchimento rápido, haverá aumento ou diminuição do tempo de desaceleração, respectivamente (LESTER et al., 2008).

O tempo de relaxamento isovolumétrico (TRIV) é outra medida obtida por meio do estudo Doppler, combinando-se a avaliação dos fluxos mitral e aórtico. Posicionando o cursor do Doppler pulsado entre os dois fluxos, é possível identificar o período entre o final da ejeção aórtica e o início do enchimento ventricular, o que corresponde ao intervalo entre o fechamento da válvula aórtica e abertura da mitral. O TRIV em cães saudáveis varia entre 38 e 54 milissegundos, sendo essa variável alterada pelo relaxamento reduzido do VE ou aumento da pressão no átrio esquerdo, fatores que irão prolongar ou diminuir o TRIV, respectivamente (BONAGURA \& SCHOBER, 2009; BOON 2011) (Figura 2).

O estudo de SCHOBER et al. (2010) propôs a utilização da razão E:TRIV para identificação de pacientes cardiopatas em insuficiência cardíaca. Eles argumentam que ao combinar o pico de velocidade $\mathrm{E}$ mitral, variável influenciada pela pressão de enchimento e relaxamento, com a variável TRIV que é mais dependente do relaxamento, o efeito do relaxamento sobre o pico de velocidade $E$ é minimizado, avaliando-se dessa maneira a pressão de enchimento ventricular. Como 
o aumento da pressão de enchimento é a alteração hemodinâmica mais presente na insuficiência cardíaca, espera-se que haja aumento do pico de velocidade $\mathrm{E}$ com diminuição do TRIV. Consequentemente, animais com doença cardíaca descompensada irão apresentar razão E:TRIV maior que cardiopatas compensados. No estudo de SCHOBER et al. (2010), por exemplo, em cães com mixomatose da válvula mitral compensada a razão E:TRIV foi de 1,74 e no grupo com MVM descompensada essa razão foi de 3,71.

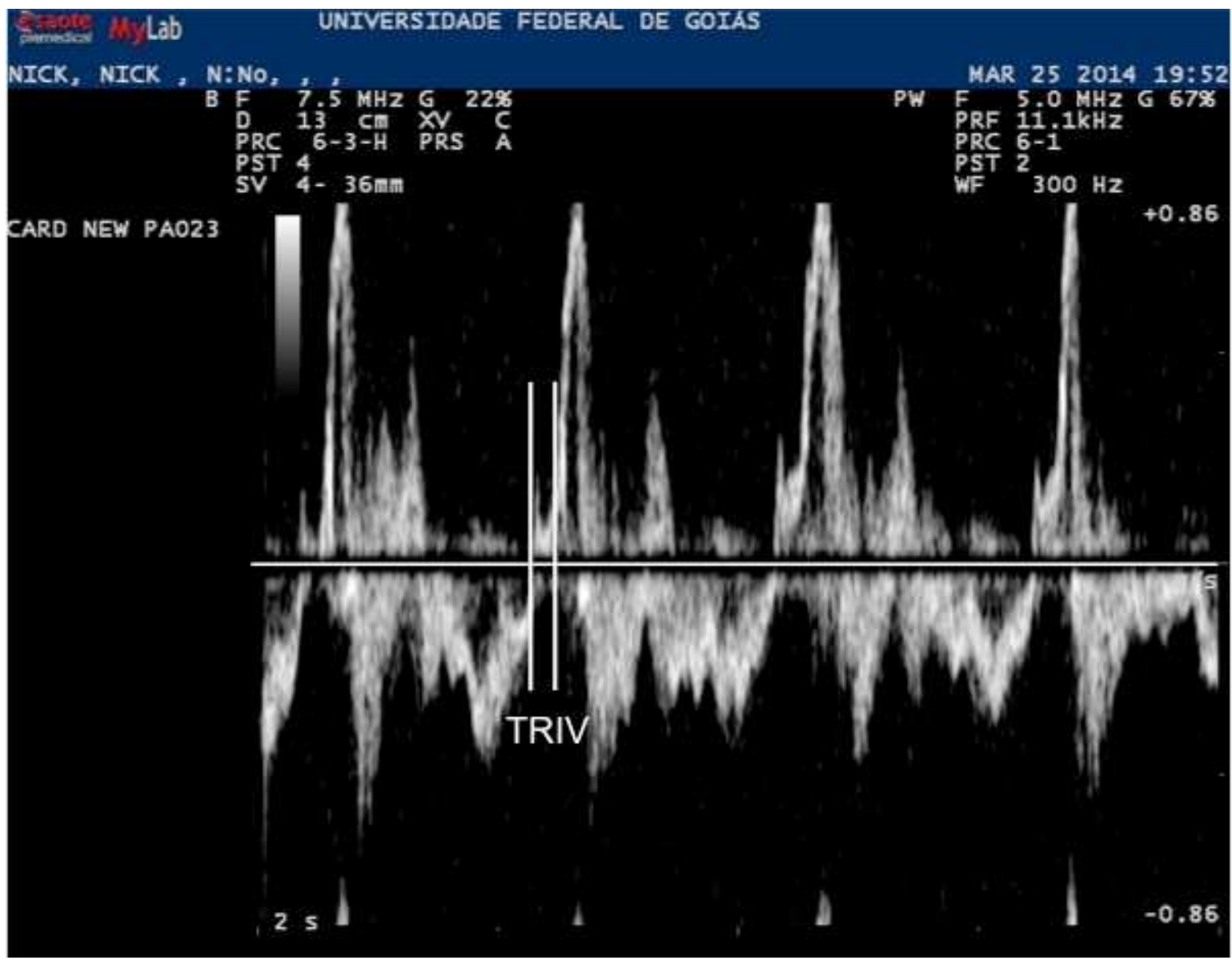

FIGURA 2. Tempo de relaxamento isovolumétrico (TRIV) de um cão normal, identificado entre barras, correspondente ao período de fechamento da válvula aórtica e abertura da mitral. Fluxo obtido posicionando-se o curso do Doppler pulsado entre o fluxos aórtico e mitral na imagem apical, paraesternal direita, cinco câmaras. Fonte: arquivo Serviço de Cardiologia Veterinária do Hospital Veterinário da UFG, 2014.

\section{Fluxo venoso pulmonar}

O padrão típico do fluxo venoso pulmonar, acessado por meio do Doppler pulsado, são duas ondas anterógradas, ondas $S$ (sistólica) e $D$ (diastólica) e uma retrógada, onda AR ("atrial reversal"- contração atrial), abaixo da linha de base. A primeira onda anterógrada, ou onda $S$, está relacionada ao declínio da pressão no átrio esquerdo durante o relaxamento atrial após a sístole ventricular. Nesse momento, estabelece-se um gradiente pressórico entre as veias pulmonares e o $A E$, ocorrendo a formação de uma onda sistólica, anterógrada (Figura 3) (PARASHAR, 
2009).

A segunda onda anterógrada, ou onda $\mathrm{D}$, ocorre durante a abertura da válvula mitral, o que irá provocar queda da pressão atrial e a formação de outra onda, dessa vez de origem diastólica. Durante a contração atrial, que ocorre ao final da diástole, o gradiente pressórico entre as veias pulmonares e átrio esquerdo se inverte, provocando a formação de uma onda retrógrada, ou onda $A R$, devido a refluxo de sangue para as veias pulmonares (Figura 3) (PARASHAR, 2009; BOON, 2011).

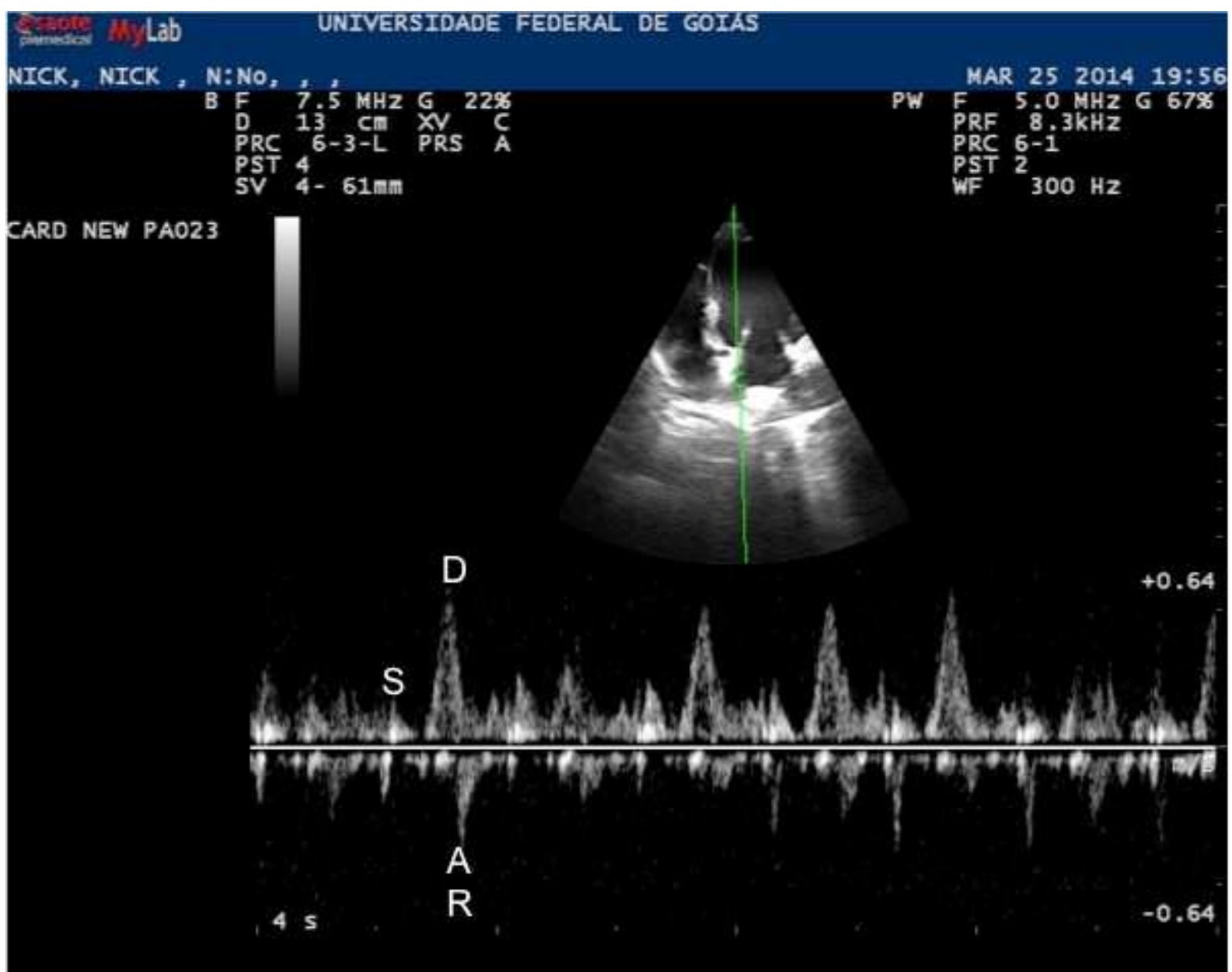

FIGURA 3. Fluxo venoso pulmonar normal de um cão, identificando picos de velocidades, das ondas S (sistólica), D (diastólica) e AR (fluxo reverso). Fonte: arquivo Serviço de Cardiologia Veterinária do Hospital Veterinário da UFG, 2014.

A razão entre a duração da onda $A$ do fluxo transmitral e da onda $A R$ do fluxo venoso pulmonar está correlacionada à pressão de enchimento do ventrículo esquerdo e a complacência desse. Mesmo em animais em que há a fusão parcial das ondas $\mathrm{E}$ e $\mathrm{A}$ do fluxo transmitral essa correlação é válida (SCHOBER et al., 2003). A avaliação do fluxo venoso pulmonar é utilizada em conjunto com o fluxo transmitral para diferenciar o grau II da disfunção diastólica ou pseudonormalização, que será discutido adiante, utilizando a relação entre as durações das ondas $A$ do fluxo mitral e AR (BONAGURA \& SCHOBER, 2009).

No entanto, vale ressaltar que em cães que apresentam regurgitação mitral grave, a avaliação do fluxo venoso pulmonar pode ser confusa devido ao refluxo de sangue para as veias pulmonares (BONAGURA \& SCHOBER, 2009). A 
fibrilação atrial é outra condição que dificulta a interpretação do fluxo venoso pulmonar, porque a perda da contração e do relaxamento atrial reduzem o fluxo venoso pulmonar sistólico, independente das pressões de enchimento (NAGUEH et al., 2009).

Outra ressalva a ser considerada é que a introdução de métodos mais avançados e sensíveis em identificar anormalidades de relaxamento do ventrículo esquerdo, como o Doppler tecidual, tem minimizado o uso do estudo do padrão de fluxo venoso pulmonar para avaliação de disfunção diastólica (LESTER et al., 2008), uma vez que esse também é de difícil execução (NAGUEH et al., 2009).

\section{Doppler Tecidual}

O estudo Doppler tecidual do miocárdio permite o acesso, não invasivo, a velocidade de movimentação do músculo cardíaco. O princípio físico do Doppler tecidual é o mesmo do Doppler pulsado convencional, no entanto, ao passo que o Doppler pulsado convencional utiliza a movimentação das hemácias para calcular a velocidade do fluxo sanguíneo em determinado vaso ou câmara cardíaca, o Doppler tecidual é calibrado para eliminar os sinais de baixa amplitude e alta velocidade das células sanguíneas e apenas detectar o sinal Doppler de alta amplitude e baixa velocidade oriundo da movimentação miocárdica (BOON, 2011; BONAGURA \& FUENTES, 2015).

Três modos de Doppler tecidual estão disponíveis, sendo os modos pulsado e colorido 2-D os mais utilizados. O modo de Doppler tecidual pulsado fornece informações sobre a movimentação miocárdica através de um único volume de amostra, sendo o cursor do Doppler posicionado sobre a área de interesse no miocárdico. Com o modo-M colorido, velocidades do miocárdio são analisadas ao longo de uma única linha de exploração selecionada, que é dirigida pelo operador da mesma maneira que para o modo $\mathrm{M}$ transventricular convencional. Este método é usado para analisar o movimento radial do septo interventricular (SIV) ou da parede livre do VE. Usando o modo Doppler tecidual colorido em 2D, em tempo real, o Doppler colorido é sobreposto à escala de cinza das imagens em 2D. Software específico é então utilizado, off line, para quantificar as velocidades ao longo do ciclo cardíaco do miocárdio em diferentes segmentos (CHETBOUL, 2010).

Tanto o Doppler tecidual colorido 2-D quanto o pulsado fornecem informações com resolução temporal e espacial suficientes para monitorar pequenas mudanças nas funções sistólica ou diastólica do miocárdio. No entanto, um estudo conduzido por WESS et al. (2010), comparando as duas técnicas em cães saudáveis, encontrou diferença estatística e clinicamente significativa entre as medições de velocidade. Embora a correlação entre os dois métodos tenha sido significativa, medidas derivadas do Doppler tecidual pulsado foram aproximadamente $2,20 \mathrm{~m} / \mathrm{s}$ mais elevadas do que os valores do Doppler colorido 2D. Isso significa que valores de referência diferentes devem ser usados para ambos os métodos.

A avaliação em tempo real da velocidade de movimentação de determinado setor do miocárdio deve ser feita obtendo-se um traçado espectral relativo à movimentação miocárdica, seja empregando o Doppler tecidual pulsado ou colorido 2-D. Assim como na avalição da movimentação das hemácias, ao estudo Doppler tecidual, o registro da movimentação miocárdica é feito em uma escala gráfica, onde o eixo das abcissas $(x)$ representa o tempo decorrido e o eixo das ordenadas $(\mathrm{y})$ representa a velocidade registrada em centímetros por segundo 
(cm/s) (BOON, 2011; BONAGURA \& FUENTES, 2015).

Ao se posicionar o cursor sobre uma área do miocárdio em um corte transversal, ou eixo curto do coração, obtém-se informação relativa a velocidade de movimentação das fibras miocárdicas circunferenciais ou radiais, ao passo que utilizando o corte longitudinal, ou eixo longo do coração, analisa-se a velocidade de movimentação das fibras longitudinais. A área mais utilizada na análise da função diastólica é o corte longitudinal, na altura do anel mitral (BOON, 2011; BONAGURA \& FUENTES, 2015).

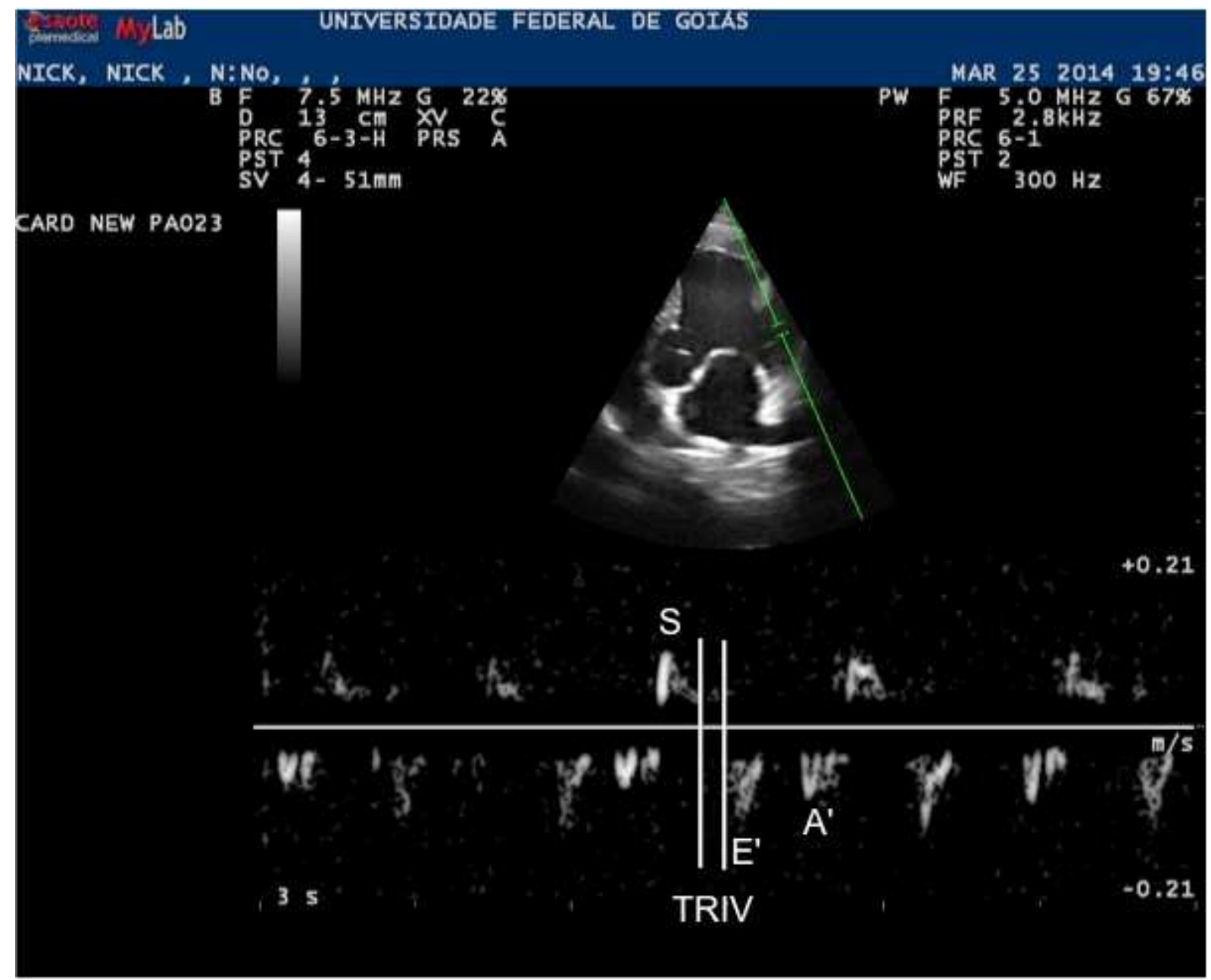

FIGURA 4. Doppler tecidual do anel mitral identificando o tempo de relaxamento isovolumétrico (TRIV), velocidades diastólicas precoce E' e tardia A'. Fonte: arquivo Serviço de Cardiologia Veterinária do Hospital Veterinário da UFG, 2014.

O perfil do traçado obtido no estudo Doppler tecidual inclui uma onda positiva sistólica denominada de $S$, após uma breve fase de contração isovolumétrica, e duas ondas negativas, denominadas E' e A', das fases diastólicas de enchimento rápido e de contração atrial, respectivamente. Entre as ondas de origem sistólica e as diastólicas é possível identificar o tempo de relaxamento isovolumétrico ventricular. Frequências cardíacas altas também irão provocar sobreposição das ondas E' e A', assim como ocorre com as ondas E e A do fluxo transmitral (PARASHAR, 2009; BOON, 2011) (Figura 4).

$A$ velocidade da onda E' é influenciada pelo relaxamento do ventrículo esquerdo, função sistólica e pressão intraventricular esquerda (BOON, 2011). A ENCICLOPÉDIA BIOSFERA, Centro Científico Conhecer - Goiânia, v.11 n.22; p.2243 2015 
velocidade da onda A' por sua vez, é determinada pela função sistólica do átrio esquerdo e pressão intraventricular esquerda, ao final da diástole. Assim sendo, um aumento da contratilidade do átrio esquerdo aumenta a velocidade da onda A', enquanto um aumento da pressão intraventricular esquerda ao final da diástole irá diminuí-la. A razão entre as velocidades das ondas E' e A' é utilizada para estimar a pressão de enchimento do VE. Uma vantagem do Doppler tecidual sobre o Doppler convencional é que ele pouco depende da pré-carga (NAGUEH et al., 2009; PARASHAR, 2009). BOON (2011) relata que em cães normais, a onda E' deve possuir velocidade superior à $7,2 \mathrm{~cm} / \mathrm{s}$. A razão entre a onda $E$ de origem mitral e a E' da movimentação miocárdica é outro parâmetro utilizado para estimar a pressão de enchimento do ventrículo esquerdo, corrigindo o efeito da pré-carga sobre a onda E do fluxo mitral, sendo que em cães normais essa razão deve ser menor que 8,07.

Em um modelo canino de desenvolvimento de disfunção diastólica, induzida por estimulação rápida do ventrículo por marcapasso, foi detectado que os parâmetros do Doppler tecidual foram mais sensíveis em detectar a disfunção diastólica, ocorrendo diminuição da onda E' 12 horas após o início da estimulação. Já as variáveis do Doppler convencional do fluxo mitral alteraram-se somente a partir do terceiro dia. Além disso, a condição de taquicardia imposta aos animais dificultou a diferenciação das ondas E e A mitral e mensuração do tempo de desaceleração (WU et al., 2008).

\section{DISFUNÇÃO DIASTÓLICA}

A disfunção diastólica é um aumento anormal da pressão diastólica final do VE para um determinado volume diastólico final. Como tal a disfunção diastólica pode ser o resultado de uma anormalidade ativa (relaxamento) ou passiva (complacência ou rigidez) do miocárdio ou ambas. Normalmente, há um aumento da pressão de enchimento do VE, que é praticamente sinônimo de pressão aumentada no AE (PARASHAR, 2009).

A disfunção diastólica é classificada na medicina em três graus ou estágios, quais sejam: Grau I ou relaxamento ventricular anormal, Grau II ou pseudonormalização, Grau III ou padrão restritivo (LESTER et al., 2008; OH et al., 2011), sendo essa mesma classificação também utilizada na medicina veterinária (KIBAR et al., 2009; SCHOBER et al.; 2010; BOON, 2011; BONAGURA \& FUENTES, 2015).

\section{Grau I ou relaxamento ventricular anormal}

O relaxamento ventricular anormal é responsável por reduzir o transporte de sangue do átrio para o ventrículo esquerdo, o que fará com que a pressão do VE permaneça alta no início da diástole, aumentado o tempo de enchimento ventricular nessa fase e também fazendo com que a contribuição da contração atrial para o preenchimento do VE seja maior. Essas mudanças no fluxo sanguíneo na entrada do VE irão refletir no fluxo mitral, à avaliação ecodopplercardiográfica (LESTER et al., 2008; BOON, 2011).

O gradiente pressórico atrioventricular na fase inicial da diástole é menor em pacientes com distúrbio de relaxamento do miocárdio, diminuindo a velocidade da onda $E$ e prolongando o tempo de desaceleração dessa, uma vez que o VE irá demorar mais para relaxar e o equilíbrio pressórico atrioventricular levará mais tempo para ocorrer. A velocidade da onda A por sua vez, estará aumentada, tornando a razão E:A menor do que um, devido a maior contribuição da contração 
atrial para o enchimento ventricular. O prolongamento do tempo de relaxamento isovolumétrico também é esperado nessa fase (BOON, 2011; OH et al., 2011). A redução do relaxamento do miocárdio reflete-se também na velocidade de movimentação do anel mitral, avaliada por meio do Doppler tecidual, provocando redução da velocidade E' (LESTER et al., 2008)(Figura 5).

Os distúrbios de relaxamento do miocárdio são usualmente encontrados em animais com cardiomiopatia hipertrófica e hipertensão, assim como em paciente geriátricos, sendo portanto, um padrão "normal" entre os idosos, uma vez que esses apresentam modificações estruturais que culminam em comprometimento do relaxamento ventricular (BOON 2011; OH et al., 2011). No estudo de KOFFAS et al. (2006) por exemplo, no qual foram avaliados por meio de Doppler tecidual gatos normais e portadores de cardiomiopatia hipertrófica, a idade dos animais normais demonstrou uma relação inversa a velocidade da onda $E$ ' e consequentemente a razão E':A'.

O relaxamento ventricular também está comprometido em cães obesos como observado por MEHLMAN et al. (2013). Nesse estudo encontraram aumento do tempo de relaxamento isovolumétrico em $37 \%$ dos animais avaliados e outros $47 \%$ demonstraram aumento da relação E:TRIV sem aumento do TRIV. Isso demonstra que os cães obesos apresentam tanto distúrbio de relaxamento, observado pelo TRIV prolongado, quanto alteração na complacência ventricular, visto que uma relação E:TRIV aumentada reflete aumento de pressão de enchimento do ventrículo esquerdo.

A cardiomiopatia hipertrófica $(\mathrm{CMH})$ é a doença cardiovascular mais comum entre os felinos, sendo as alterações mais apontadas ao estudo Doppler tecidual a diminuição da onda E', aumento da onda A' e consequente inversão da razão $E^{\prime}: A^{\prime}$ e aumento do tempo de relaxamento isovolumétrico (KOFFAS et al., 2006; SAMPEDRANO et al., 2006). As alterações do padrão do fluxo mitral incluem diminuição da onda E e razão E:A (SAMPEDRANO et al., 2006).

SAMPREDRANO et al. (2006) relataram presença de disfunção diastólica em um grupo de gatos com $\mathrm{CMH}$ sem sinais de hipertrofia ao modo-M do exame ecocardiográfico bidimensional, a não ser na porção subaórtica do septo interventricular, encontrando disfunção diastólica nas outras regiões do coração aparentemente "normais", como a parede livre do VE. Essa mesma situação também foi reportada em um grupo de cães com hipertensão sistêmica e que ainda não apresentava alterações sugestivas de hipertrofia ao modo- $M$, consequência comum da enfermidade, porém apresentou inversão da razão E'/A' do estudo Doppler tecidual (MISBACH et al., 2011).

Uma consequência comum da hipertensão sistêmica, tanto em cães como em gatos, é o desenvolvimento de hipertrofia concêntrica do ventrículo esquerdo. Estudos realizados em cães (MISBACH et al., 2011) e gatos hipertensos (SAMPEDRANO et al., 2006) relataram alterações de função diastólica semelhantes as encontradas em felinos portadores de $\mathrm{CMH}$, citadas anteriormente, sendo que 0 estudo de SAMPEDRANO et al. (2006) não encontrou diferença entre os gatos com $\mathrm{CMH}$ e o grupo de felinos hipertensos. 


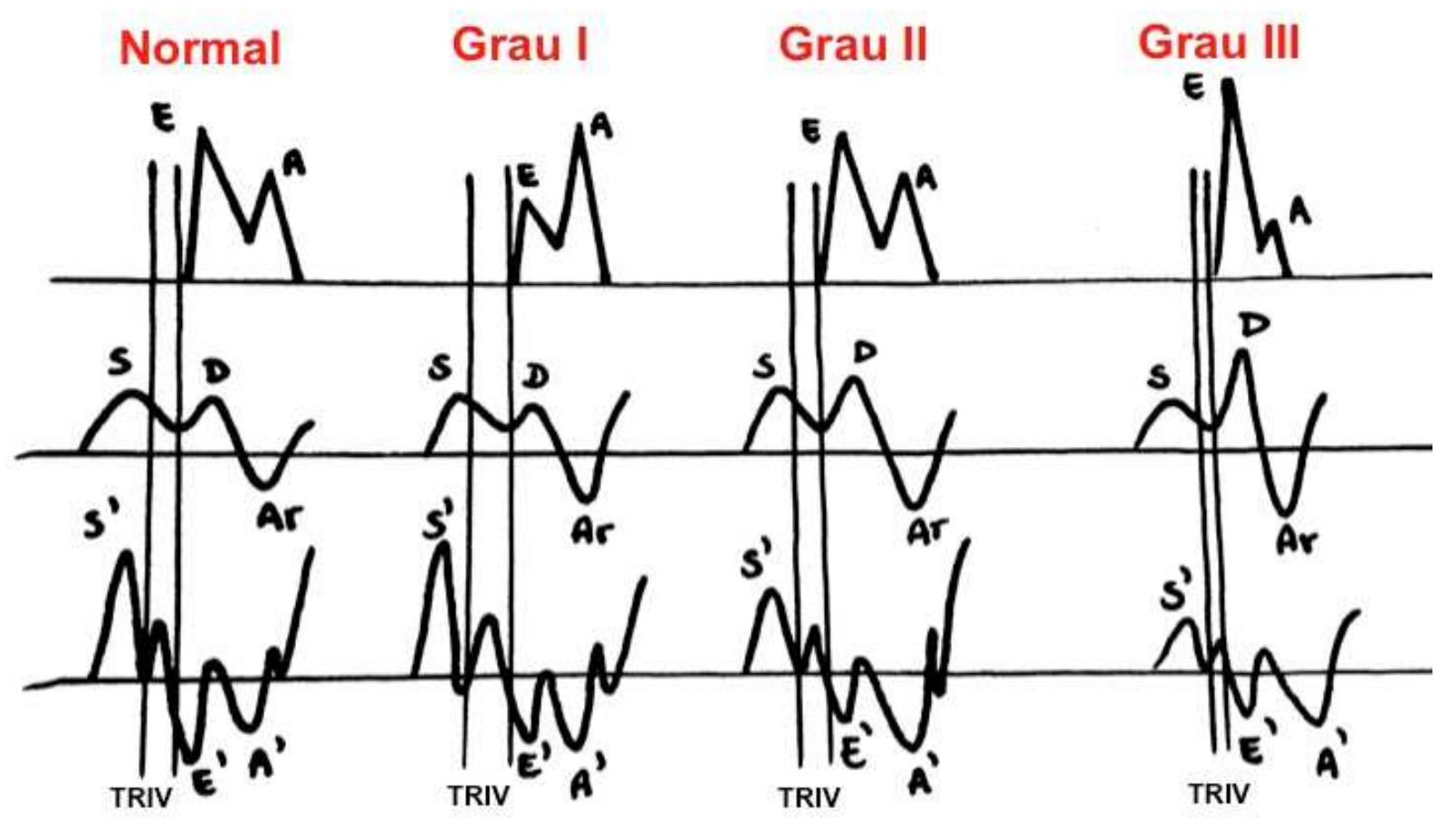

FIGURA 5: Diagrama demonstrando a progressão desde uma função diastólica preservada até o grau III ou restritivo, acessada por meio da avaliação do fluxo transmitral (primeira linha), fluxo venoso pulmonar (segunda linha) e doppler tecidual (terceira linha). $E=$ pico da onda $E$ mitral. $A=$ pico da onda $A$ mitral, $S=$ velocidade sistólica da veia pulmonar, $D=$ velocidade diastólica da veia pulmonar, $\mathrm{Ar}=$ fluxo reverso atrial da veia pulmonar, S'= velocidade de movimentação sistólica do miocárdio; E'= velocidade de movimentação diastólica da fase de enchimento rápida; $A^{\prime}=$ velocidade de movimentação diastólica da fase de contração atrial.

\section{Grau II ou pseudonormalização}

O grau II, ou pseudonormalização, é assim denominado porque a razão $\mathrm{E}: \mathrm{A}$, antes alterada durante a fase de distúrbio do relaxamento miocárdico, parece normal. Esse padrão está presente em casos onde há comprometimento do relaxamento do miocárdio, porém com complacência preservada, associado ao aumento da pressão no átrio esquerdo, que por sua vez estará aumentada devido à restrição ao esvaziamento atrial. A medida que a pressão do átrio esquerdo aumenta com o avanço da disfunção diastólica, a velocidade diastólica inicial ou onda $\mathrm{E}$ consequentemente aumentará (LESTER et al., 2008; PARASHAR, 2009; BOON, 2011).

Durante o período em que a pressão atrial é alta o bastante para retornar a aparência do fluxo mitral ao normal, o tempo de desaceleração também estará normal porque o tempo para que a pressão ventricular caia abaixo da pressão atrial estará reduzido. Contrariamente, a velocidade E' permanecerá reduzida, identificando a anormalidade de relaxamento do ventrículo esquerdo. A diminuição da onda E' e aumento da onda E mitral, aumenta a razão E/E', sendo esse dado, associado ou não ao achado de aumento de $\mathrm{AE}$, utilizado para diferenciar paciente 
com função diastólica preservada, daqueles em padrão de pseudonormalização (LESTER et al., 2008; BOON, 2011) (Figura 5).

KIBAR et al. (2009) objetivaram em seu estudo explorar a execução e valor diagnóstico do Doppler tecidual e ecodopplercardiografia convencional na determinação da disfunção diastólica em cães, relatando que os parâmetros do fluxo transmitral isolados ( $E, A, E / A$ e $D T)$ não auxiliaram na diferenciação entre os grupos: normal e pseudonormal. No entanto, à avaliação Doppler tecidual, observaram aumento da onda $A^{\prime}(>7,6 \mathrm{~cm} / \mathrm{s})$ e diminuição razão $E^{\prime} / A^{\prime}$, sendo que esses animais apresentavam sintomas de disfunção cardíaca com função sistólica preservada. Utilizando os achados de onda A'> 7,6cm/s e razão E/E'> 12,34 como valores de corte para identificação do padrão de pseudonormalização, eles obtiveram sensibilidade de $83 \%$ e especificidade de $79 \%$, concluindo que a associação de achados ecodopplercardiográficos convencionais e de Doppler tecidual são imprescindíveis para avaliação da disfunção diastólica em cães.

O fluxo venoso pulmonar para o átrio esquerdo é outra avaliação que pode ser empregada para identificação do Grau II da disfunção diastólica. O aumento da pressão atrial está relacionado ao aumento da velocidade e duração do fluxo retrógrado AR para as veias pulmonares, podendo a duração da onda AR ser maior que a duração da onda $A$ mitral, diminuindo a razão entre as durações das ondas A:AR. Como a pressão atrial está levada espera-se encontrar diminuição da onda S e aumento da onda D, reduzindo a razão S/D (PARASHAR, 2009) (Figura 5).

A pseudonormalização foi a anormalidade diastólica mais frequente nos 76 boxers com estenose aórtica avaliados no estudo de SCHOBER \& FUENTES (2002), sendo encontrada mesmo em cães com estenose aórtica leve. Para diferenciar o grau II da disfunção diastólica de um fluxo mitral normal, eles utilizaram outros parâmetros ecocardiográficos que não o fluxo transmitral, quais sejam, achado de hipertrofia do VE ou dilatação do $A E$ e avaliação do fluxo venoso pulmonar, mais especificamente, aumento da duração do fluxo venoso pulmonar reverso AR, devido ao aumento da pressão atrial. O grau I da disfunção diastólica foi o segundo mais observado nos cães com estenose aórtica, refletindo o distúrbio de relaxamento encontrado em ventrículos hipertrofiados devido ao aumento de pós carga imposta pela estenose no fluxo de saída do VE.

\section{Grau III ou padrão restritivo}

O padrão restritivo ou grau III é considerado o pior e mais avançado grau de disfunção diastólica (PARASHAR, 2009). Esse tipo de disfunção diastólica está presente em pacientes com cardiomiopatias restritiva, dilatada, hipertrófica ou isquêmica, condições em que a pressão de enchimento do ventrículo esquerdo encontra-se alta devido a um miocárdio não complacente. $O$ aumento da pressão do átrio esquerdo associado a um ventrículo com comprometimento severo de seu relaxamento e complacência é também encontrado em padrões restritivos de enchimento (BOON, 2011).

Em outras palavras, pode-se concluir que a fisiopatogenia do desenvolvimento da disfunção diastólica grau III compreende a combinação de fatores que predispõem ao aumento de pressão no $A E$ e diminuição da complacência do VE associado a um miocárdio com comprometimento severo de seu relaxamento. $O$ aumento da pressão do $A E$ aumenta o gradiente pressórico atrioventricular, porém o ventrículo não complacente aumenta a pressão de enchimento do VE o que por sua vez, diminui o tempo gasto para que ocorra a equalização das pressões no átrio e ventrículo esquerdos, determinando uma onda 
E mitral de velocidade mais elevada e consequentemente, reduzindo o tempo de desaceleração dessa (PARASHAR, 2009) (Figura 5). Inicialmente, o átrio esquerdo em um mecanismo compensatório, aumento sua força de contração, porém com o agravamento da disfunção diastólica, estabelece-se uma disfunção atrial, diminuindo o volume de esvaziamento atrial. Em pacientes humanos já foi estabelecido que ocorre remodelamento atrial frente a uma disfunção diastólica e que o volume atrial reflete a severidade dessa disfunção (TEO et al., 2010). Em felinos portadores de $\mathrm{CMH}$, doença reconhecida por cursar com comprometimento diastólico, foi observada também disfunção atrial (LINNEY et al., 2014).

Nessa situação, como a maior parte do enchimento ventricular ocorre no início da diástole, e o átrio esquerdo encontra-se relativamente vazio, menos sangue será bombeando na fase de enchimento ventricular devido a contração atrial, o que é refletido em uma onda $A$ de baixa velocidade e consequentemente razão $E: A$ maior que dois (PARASHAR, 2009; OH et al., 2011). O TRIV pode estar reduzido ou normal em corações com padrão restritivo de enchimento (BOON, 2011).

O fluxo venoso pulmonar também encontra-se alterado frente a uma pressão atrial elevada, sendo observada diminuição da velocidade sistólica e aumento da duração e velocidade do fluxo reverso pulmonar AR. Um fluxo reverso pulmonar de duração maior que a onda $A$ mitral é indicativo de diminuição da complacência ventricular, assim como de pressão de enchimento ventricular elevada (BOON, 2011).

À semelhança do que ocorre nas outras fases da disfunção diastólica, a onda E' referente à velocidade de movimentação do miocárdio estará reduzida, porém em maior proporção e, observa-se também, redução da onda A' em consequência de uma disfunção do átrio, que na maioria dos casos encontra-se dilatado $(\mathrm{OH}$ et al., 2011). Consequentemente a razão E:E' estará aumentada, uma vez que a velocidade da onda $E$ encontra-se elevada e esse achado é utilizado para indicar aumento da pressão de enchimento do VE (BOON, 2011).

Em linhas gerais, avaliando-se os padrões do fluxo mitral e movimentação do anel mitral ao Doppler tecidual, desde uma função diastólica preservada até o grau mais severo de disfunção diastólica, percebe-se que a onda $E$ de origem mitral é extremamente sensível a pré-carga, tornando-se mais alta com diminuição do tempo de desaceleração com a piora da função diastólica e aumento da pressão de enchimento. Já a onda E' originada da movimentação miocárdica é menos sensível as condições de pré-carga e reduz-se em todos os estágios de disfunção diastólica, refletindo as alterações de relaxamento do miocárdio, sendo sua redução usualmente reportada como a manifestação mais precoce de distúrbio diastólico $(\mathrm{OH}$ et al., 2011).

O estudo de O'SULLIVAM et al. (2007) demonstrou que em cães com cardiomiopatia dilatada (CMD) o grau de disfunção diastólica é correspondente ao quadro clínico apresentado pelo animal uma vez que Dobermans com CMD, oculta ou sem sinais clínicos, apresentaram, em sua maioria, pseudonormalização do fluxo mitral ao passo que, nos animais com CMD evidente, o padrão restritivo ou grau III da disfunção diastólica foi o mais encontrado, caracterizado por razão $E: A>2$ e redução do tempo de desaceleração e das velocidades E' e A'. Nesse mesmo estudo, as variáveis ecodopplercardiográficas foram avaliadas quanto aos seus valores prognósticos e foi observado que a redução do tempo de desaceleração foi significativamente associada ao tempo de aparecimento da insuficiência cardíaca e morte súbita em cães com CMD oculta.

Similarmente, o estudo de SCHOBER et al. (2010) demonstrou que cães 
na fase pré-clínica da CMD possuem evidências ecodopplercardiográficas de função diastólica preservada ou Grau I da disfunção, enquanto animais com CMD evidente apresentaram o Grau III. Em contrapartida, essa progressão da disfunção diastólica, acompanhando o desenvolvimento da cardiopatia, foi menos evidente em paciente portadores de mixomatose da válvula mitral (MVM).

TIDHOLM et al. (2009) também verificaram que os achados ecodopplercardiográficos de cães com MVM diferem daqueles apresentados por cães portadores de outras enfermidades cardiovasculares, que não cursam com regurgitação mitral. Ao passo que observaram comprometimento diastólico ao estudo Doppler do fluxo mitral, encontrando aumento da velocidade das ondas $E$ e da razão $E: A$ em cães com MVM e sinais de insuficiência cardíaca. O estudo Doppler tecidual, entretanto, demonstrou contrariamente, melhora da função diastólica com aumento da velocidade da onda E' do anel mitral. Esse padrão observado por TIDHOLM et al. (2009) pode ser caracterizado como "pseudorestritivo" (SCHOBER et al., 2010).

Tanto o estudo de SCHOBER et al. (2010) quanto o de TIDHOLM et al. (2009) justificam que o comportamento anormal do fluxo transmitral, em cães com MVM, deve-se à presença da regurgitação mitral. Enquanto na CMD a pressão de enchimento do VE é primariamente influenciada pela disfunção sistólica acompanhada de diminuição da complacência ventricular, em cães com MVM o aumento de pressão de enchimento do VE é em decorrência da sobrecarga de volume sanguíneo imposta por uma válvula insuficiente. Essa sobrecarga sanguínea associada a um VE com função sistólica, complacência e relaxamento preservados, diminui o valor diagnóstico do fluxo mitral que é extremamente dependente da précarga.

MASUTANI et al. (2008) compararam os achados ecodopplercardiográficos de cães com insuficiência cardíaca (IC) induzida experimentalmente e cães com funções sistólicas e diastólicas preservadas e submetidos a uma sobrecarga de volume. Similarmente ao reportado por TIDHOLM et al. (2009) em cães com regurgitação mitral, eles observaram que os cães normais submetidos a sobrecarga de volume apresentaram evidências de disfunção diastólica à avaliação do fluxo mitral, com diminuição do DT, em menor grau ao observado no grupo com IC, porém com aumento da velocidade E' ao Doppler tecidual enquanto os cães com IC apresentaram diminuição da E'. Eles então concluíram que o padrão restritivo pode ser diferenciado da sobrecarga de volume imposta a um ventrículo normal quando encontra-se concomitantemente aos achados do fluxo mitral, diminuição do DT e E', o que reflete relaxamento diminuído e aumento da rigidez ventricular.

BONAGURA \& SCHOBER (2009) e CHETBOUL \& TISSIER (2012), amparados por achados de outros estudos que reportam o comportamento não tradicional do fluxo transmitral em cães com regurgitação mitral, fizeram a ressalva de que a utilização da razão E:E', utilizada para estimar a pressão de enchimento do VE em outras cardiopatias, não se aplica nesses cães. Nos casos avançados de regurgitação mitral há, como dito anteriormente, uma sobrecarga de volume e, se a função diastólica estiver preservada, como nos animais do estudo de TIDHOLM et al. (2009), a razão E:E' apresenta pobre valor estimativo de pressão de enchimento e, justamente por isso, a estimativa de altas pressões de enchimento características de insuficiência cardíaca são mais difíceis de detectar em pacientes portadores de MVM.

SCHOBER et al. (2008) e SCHOBER et al. (2010) propuseram a 
utilização da razão E:TRIV como índice previsor da pressão de enchimento do VE, sendo até mais sensível que a razão $E$ :E' anteriormente mencionada. No estudo SCHOBER et al., (2008) demonstraram que além de possuir alta correlação com a pressão do VE, mensurada invasivamente, a razão E:TRIV foi sensível em detectar a queda na pressão diastólica final do VE quando os animais com IC experimentalmente induzida foram medicados com furosemida. Ou seja, a razão E:TRIV foi eficaz em detectar queda da pressão de enchimento e, portanto, pode ser utilizada para monitorar eficiência terapêutica.

SCHOBER et al. (2010) avaliaram os índices ecodopplercardiográficos de cães com CMD e MVM de ocorrência natural e propuserem que esses seriam capazes de prever a ocorrência de insuficiência cardíaca congestiva (ICC). Ao final do estudo e comparando os índices com o sinais radiográficos sugestivos de edema pulmonar, eles concluíram que o estabelecimento da classe de disfunção diastólica, razão E:TRIV e o TRIV foram as variáveis mais sensíveis em detectar pacientes com cardiopatia descompensada. Os resultados desse estudo comprovaram, portanto, a utilidade clínica de se acessar a função diastólica do VE em pacientes com CMD ou MVM, sendo a disfunção diastólica, particularmente de padrão restritivo altamente preditiva de quadro de ICC nesses pacientes.

\section{CONSIDERAÇÕES FINAIS}

Os métodos ecodopplercardiográficos apresentados fornecem acesso não invasivo aos padrões de enchimento do ventrículo esquerdo e, dessa forma, à função diastólica do mesmo. Como foi relatado durante essa revisão, as variáveis utilizadas para mensurar as pressões de enchimento do VE possuem grande correlação com os valores mensurados invasivamente e isso representa um grande avanço para a cardiologia veterinária porque, a maioria dos pacientes para os quais a estimativa dessa pressão é necessária para determinar seu prognóstico são cardiopatas descompensados, apresentando grande risco anestésico, inviabilizando a realização de procedimentos invasivos, como a implantação de cateteres, para aferição da pressão ventricular.

Percebe-se, no decorrer da revisão, que o Doppler tecidual representou uma evolução na detecção de animais com disfunção diastólica, permitindo identificar pacientes nas fases mais precoces, sendo mais sensível que o método Doppler pulsado convencional. Por ser mais específica em detectar alterações de relaxamento do miocárdio, a técnica Doppler tecidual complementa os achados da avaliação do fluxo transmitral por meio do Doppler convencional, sendo determinante para a detecção de pacientes na fase de pseudonormalização.

No entanto, nem todos os aparelhos ecodopplercardiográficos possuem o software necessário para execução da técnica Doppler tecidual. Essa realidade exige que o profissional tenha conhecimento dos outros índices utilizados para identificação do paciente no grau II da disfunção diastólica, como os achados da avaliação do fluxo Doppler venoso pulmonar e avaliação da dimensão atrial esquerda.

Como foi possível perceber, o estabelecimento do grau de disfunção diastólica é extremamente importante na rotina clínica da cardiologia veterinária, visto que fornece informações relativas ao prognóstico do paciente e permite monitorar o sucesso da terapêutica estabelecida e até mesmo detecta o risco de desenvolvimento de insuficiência cardíaca e morte súbita. Não obstante, vale ressaltar que os achados da avaliação ecodopplercardiográfica devem ser interpretados em conjunto com os achados clínicos e histórico do paciente, assim 
como em qualquer outro método de diagnóstico complementar, particularmente naqueles pacientes portadores de mixomatose da válvula mitral, em que o achado de um padrão "pseudorestritivo" é frequente, mesmo com função diastólica preservada.

\section{REFERENCIAS}

BONAGURA, J. D.; FUENTES, V. L. Ecocardiography. In: MATTOON, J. S.; NYLAND, T. G. Small animal diagnostic ultrasound. 3 ed. St. Luis, Missouri: Elsevier Saunder, p.217-331p.2015.

BOON, J. A. Veterinary echocardiography. 2 ed. West Sussex: Wiley Blackwell, 2011. 632p.

BONAGURA, J. D.; SCHOBER, K. E. Can ventricular function be assessed bay echocardiography in chronic canine mitral disease?. Journal of Small Animal Practice, Oxford, v. 50, suppl. 1, p. 12-24, 2009.

CHETBOUL, V. Advanced techniques in echocardiography in small animals. The Veterinary Clinics of North America. Small Animal Practice, Philadelphia, v. 40, p. 529-543, 2010.

CHETBOUL, V.; TISSIER, R. Echocardiographic assessment of canine degenerative mitral valve disease. Journal of Veterinary Cardiology, Amsterdam, v. 14, p. 127148, 2012.

KUZNETSOVA, T.; THIJS, L.; KNEZ, J.; HERBOTS, L.; ZHANG, Z.; STAESSEN, J. A. Prognostic value of left ventricular diastolic disfunction in a general population. Journal of the American Heart Association, Oxford, 3: e000789, 2014.

KIBAR, M.; MARKOVIC, M.; KOLM, U. S.; THALHAMMER, J. Determination of diastolic dysfunction by conventional and Doppler tissue echocardiography in dogs. Turkish Journal of Veterinary \& Animal Sciences, Kars, v. 33, n. 6, p. 501-507, 2009.

KOFFAS, H.; DUKES-McEWAN, J.; CORCORAN, B. M.; MORAN, C. M.; FRENCH, A.; SBOROS, V.; SIMPSON, K.; McDICKEN, W. N. Pulsed tissue Doppler imaging in normal cats and cats with hypertrophic cardiomyopathy. Journal of Veterinary Internal Medicine, Lawrence, v. 20, p. 65-77. 2006.

LESTER, S. J.; NISHIMURA, R. A.; KHANDHEIRA, B. K. Unlocking the mysteries of diastolic function: deciphering the Rosetta Stone 10 years later. Journal Of The America College of Cardiology, Washington, v. 51, n. 7, p. 679-689, 2008.

LINNEY, C. J.; DUKES-McEWAN, J.; STHEPENSON, H. M.; LOPEZ-ALVAREZ, J.; FONFARA, S. Left atrial size, atrial function and left ventricular diastolic function in cats with hypertrofic cardiomyopathy. Journal of Small Animal Practice, Oxford, v. 55, p. 198-206, 2014.

LO, Q.; THOMAS, L. Echocardiographic evaluation of diastolic heart faliure. 
Australasian Journal of Ultrasound in Medicine, Sydney, v. 13, n. 1, p. 14-26, 2010.

MASUTANI, S.; LITTLE, W. C.; HASEGAWA, H.; CHENG, H.; CHENG, C. Restrictive left ventricular filling pattern does not result from increased left atrial pressure alone. Circulation, Dallas, v.117, p.1550-1554, 2008.

MEHLMAN E.; BRIGHT, J. M.; JECKEL, K.; PORSCHE, C.; VEERAMACHANENI, D. N. R.; FRYE, M. Echocardiographic evidence of left ventricular hypertrophy in obese dogs. Journal of Veterinary Internal Medicine, Lawrence, v. 27, p. 62-68, 2013.

MISBACH, C.; GOUNI, V.; TISSIER, R.; TREHIOU-SECHI, E.; PETIT, A. M. P.; SAMPEDRANO, C. C.; POUCHELON, J. L.; CHETBOUL, V. Echocardiohraphic and tissue Doppler imaging alterations associated with spontaneous canine systemic hypertension. Journal of Veterinary Internal Medicine, Lawrence, v. 25, p. 10251035, 2011.

NAGUEH, S. F.; APPLETON, C. P.; GILLEBERT, T. C.; MARINO, P. N.; OH, J. K.; SMISETH, O. A.; PELLIKKA, P. A.; EVANGELISTA, A. Recommendations for the evaluation of left ventricular diastólica function by echocardiography. Journal of the American Society of Echocardiohraphy, Saint Louis, v. 22, n. 2, p. 107-133, 2009.

$\mathrm{OH}$, J. L.; PARK, S.; NAGUEH, S. F. Established and novel clinical applications of diastolic function assessment by echocardiography. Circulation: Cardiovascular Imaging, Dallas, v. 4, p. 444-455, 2011.

O'SULLIVAN, M. L.; O'GRADY, M. R.; MINORS, S. L. Assessment of diastolic function by doppler echocardiography in normal Doberman pinschers and Doberman pinschers with dilated cardiomyopathy. Journal of Veterinary Internal Medicine, Lawrence, v. 21, p. 81-91, 2007.

PARASHAR, S. K. Diastolic functions: evaluation \& clinical applications. Cardiovascular Journal, Bangladesh, v. 1, n. 2, p. 132-141, 2009.

SAMPEDRANO, C. C.; CHETBOUL, V.; GOUNI, V.; NICOLLE, A. P.; POUCHELON, J. L.; TISSIER, R. Systolic and diastolic myocardial dysfunction in cats with hypertrophic cardiomyopathy or systemic hypertension. Journal of Veterinary Internal Medicine, Lawrence, v. 20, p. 1106-1115, 2006.

SCHOBER, K. E.; FUENTES, V. L. Doppler echocardiographic assessment of left ventricular diastolic function in 74 boxer dogs with aostic stenosis. Journal of Veterinary Cardiology, Amsterdam, v. 4, n. 1, p. 7-16, 2002.

SCHOBER, K. E.; FUENTES, V. L.; BONAGURA, J. D. Comparison between invasive hemodynamic measurements and noninvasive assessment of left ventricular diastolic function by use of Doppler echocardiography in healthy anesthetized cats. American Journal of Veterinary Research, Chicago, v. 64, p. 93-103, 2003.

SCHOBER, K. E.; STERN, J. A.; DACUNHA, D. N. Q. T.; PEDRAZA-TOSCANO, A. M.; SCHEMANSKI, D.; HAMLIN, R. L. Estimation of left ventricular filling pressure by ENCICLOPÉDIA BIOSFERA, Centro Científico Conhecer - Goiânia, v.11 n.22; p.2252 2015 
Doppler echocardiographic in dog with pacing-induced heart faliure. Journal of Veterinary Internal Medicine, Lawrence, v. 22, p. 578-585, 2008.

SCHOBER, K. E.; HART, T. M.; STERN, J. A.; LI, X., SAMII, V. F., ZEKAS, L. J.; SCANSEN, B. A.; BONAGURA, J. D. Detection of congestive heart failure in dogs by Doppler echocardiography. Journal of Veterinary Internal Medicine, Lawrence, v. 24, p. 1358-1368, 2010.

STALMANS, I; VANDEWALLE, E.; ANDERSON, D. R.; COSTA, V. P.; FRENKEL, R. E. P.; GARHOFER, G.; GRUNWALD, J.; GUGLETA, K.; HARRIS, A.; HUDSON, C.; JANULEVICIENE, I.; KAGEMANN, L.; KERGOAT, H.; LOVASIK, J. V.; LANZL, I.; MARTINEZ, A.; NGUYEN, Q. D.; PLANGE, N.; REITSAMER, H. A.; SEHI, M.; SIESKY, B.; ZEITZ, O.; ORGÜL, S.; SCHMETTERER, A. Use of colour Doppler imaging in ocular blood flow research. Acta Ophtalmologica, Hvidovre, v. 89, n. 8, p. e609-e630, 2011.

TEO, A. G.; YANG, H.; CHAI, P.; YEO, T. C. Impact of left ventricular diastolic disfunction on left atrial volume and function: a volumetric analysis. European Journal of Echocardiography, Amsterdan, v. 11, p. 38-43, 2010.

TIDHOLM, A.; LJUNGVALL, I.; HOGLUND, K.; WESTLING, A. B.; HAGGSTROM, J. Tissue Doppler and strain imaging in dogs with myxomatous mitral valve disease in differente stages of congestive heart failure. Journal of Veterinary Internal Medicine, Lawrence, v. 23, p. 1197-1207, 2009.

WESS, G.; KILLICH, M.; HARTMANN, K. Comparison of pulsed wave and color Doppler myocardial velocity imaging in healthy dogs. Journal of Veterinary Internal Medicine, Lawrence, v. 24, p. 360-366, 2010.

WU, W.; WANG, H.; TANG, Y.; YUAN, W.; WANG, H.; JIANG, Y. Application of quantitative tissue velocity imaging to evaluate left ventricular early diastolic dysfunction in dogs with heart failure due to rapid ventricular pacing. Journal of the American Society of Echocardiography, Saint Louis, v. 21, n. 11, p. 1269-1276, 2008. 